\title{
LOCAL ENDOMORPHISM NEAR-RINGS
}

\author{
by CARTER G. LYONS and GARY L. PETERSON
}

(Received 6th July 1987)

The purpose of this paper is to study the consequences of an endomorphism near-ring of a finite group being a local near-ring and the existence of such near-rings. As we shall see in Section 2, an endomorphism near-ring of a finite group being local gives us some information about both the structure of the group (Theorem 2.2) and the automorphisms of the group lying in the near-ring (Theorem 2.3). Existence of local endomorphism near-rings of finite groups is considered in Section 3 where we obtain as our main result that any p-group of automorphisms of a p-group containing the inner automorphisms always generates a local endomorphism near-ring. In particular, we get as a corollary that the endomorphism near-ring of a finite group $G$ generated by the inner automorphisms of $G$ is local if and only if $G$ is a p-group. The third section concludes with a discussion of endomorphism near-rings of dihedral 2-groups and generalized quaternion groups.

\section{Preliminaries}

We shall follow the conventions of [7] with regard to notation and terminology, while our basic reference on local near-rings is [6] suitably modified to the aforementioned specifications. In particular, this means that a near-ring $R$ is local if the set

$$
L=\{r \in R \mid r \text { does not have a right inverse }\}
$$

is a right $R$-subgroup of $R$.

Throughout, $G$ will denote a finite group written additively (but not necessarily abelian). The sets of inner automorphisms, automorphisms, and endomorphisms of $G$ will be denoted $\operatorname{Inn}(G), \operatorname{Aut}(G)$, and $\operatorname{End}(G)$, respectively. If $S$ is a semigroup of endomorphisms of $G, S$ generates the endomorphism near-ring $R$ under pointwise addition and composition of functions which is a distributively generated (d.g.) nearring. The endomorphism near-rings generated by $\operatorname{Inn}(G), \operatorname{Aut}(G)$, and $\operatorname{End}(G)$ will be respectively denoted $I(G), A(G)$, and $E(G)$.

As in [7], we shall say that an endomorphism near-ring $R$ generated by a semigroup of endomorphisms $S$ is tame when $\operatorname{Inn}(G) \leqq S$. The reader should keep in mind that the notion of a subgroup $H$ of $G$ being an $R$-submodule (or $R$-subgroup) coincides with the notion of $H$ being an $R$-ideal when $R$ is tame [7, Lemma 10.7]. Moreover, it is easy to see that this equivalence extends to quotients of $G$ by $R$-submodules: 
Lemma 1.1. If $R$ is a tame endomorphism near-ring of $G, H$ is an $R$-ideal of $G$, and $\bar{G}=G / H$, then a subgroup $\bar{K}$ of $\bar{G}$ is an $R$-submodule if and only if $\bar{K}$ is an $R$-ideal of $\bar{G}$.

Finally, the standard radicals $\left(J_{2}(R), J_{1}(R)\right.$, and $\left.J_{0}(R)\right)$ are all the same when $R$ is a tame endomorphism near-ring and we shall denote the radical in this setting by $J(R)$.

If $H$ and $K$ are subgroups of a group $G$, we will use $[H, K]$ to denote the subgroup of

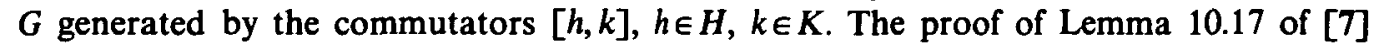
can be easily modified to obtain:

Lemma 1.2. Let $(R, S)$ be a d.g. near-ring and $G$ be an $(R, S)$-group. If $H$ and $K$ are $(R, S)$-subgroups of $G$, then $[H, K]$ is an $(R, S)$-subgroup of $G$.

In particular, we have that commutators of $R$-ideals in $G$ are $R$-ideals when $R$ is a tame endomorphism near-ring of $G$.

From time to time we will make use of the following notions from group theory: An element $\alpha$ in $\operatorname{Aut}(G)$ is said to stabilize a series

$$
0=G_{0} \leqq G_{1} \leqq G_{2} \leqq \cdots \leqq G_{n}=G
$$

of subgroups of $G$ if $\left(g+G_{i}\right) \alpha=g+G_{i}$ for all $g \in G_{i+1}$ for all $i=0,1, \ldots, n-1$. In this case, $1-\alpha$ annihilates the series and is nilpotent of degree $n$ in any endomorphism near-ring containing 1 and $\alpha$. A subgroup $A$ of $\operatorname{Aut}(G)$ is called a stability group of $G$ if there is a series of subgroups

$$
0=G_{0} \leqq G_{1} \leqq \cdots \leqq G_{n}=G
$$

stabilized by each element of $A$. Using $\pi(G)$ to denote the set of primes dividing $|G|$, a basic and easily proved result about stability groups is the following (or see [8, Lemma 5] which is an even better result):

Lemma 1.3. If $A$ is a stability group of $G$, then $\pi(A) \leqq \pi(G)$.

\section{Consequences of localness}

Let $R$ be a tame endomorphism near-ring of a group $G$ that is local. We begin this section by noting that $L=J(R)$ [6, Theorem 2.10] and proceed to obtain some results about $G$ and $R / L$. Let

$$
0=G_{0} \leqq G_{1} \leqq G_{2} \leqq \cdots \leqq G_{n}=G
$$

be an $R$-principal series (that is, a maximal series of $R$-submodules) of $G$.

Lemma 2.1. Let $R$ be a tame endomorphism near-ring of $G$ that is local and let $\bar{g}$ be any nonzero element of $G_{i} / G_{i-1}$. Then

$$
\operatorname{Ann}_{R}(\bar{g})=\operatorname{Ann}_{R}\left(G_{i} / G_{i-1}\right)=J(R)
$$

for all $i=1,2, \ldots, n$. 
Proof. Clearly we have

$$
\operatorname{Ann}_{R}(\bar{g}) \geqq \operatorname{Ann}_{R}\left(G_{i} / G_{i-1}\right) \geqq J(R)=L
$$

Since $L$ is the unique maximal $R$-subgroup of $R$ [6, Theorem 2.2], $L \geqq \operatorname{Ann}_{R}(\bar{g})$ and the result follows.

Theorem 2.2. If $R$ is a tame endomorphism near-ring of $G$ that is local, then:

(i) $G_{i} / G_{i-1} \simeq R / L$ as $R$-modules for $i=1,2, \ldots, n$.

(ii) $G_{i} / G_{i-1}$ is an elementary abelian p-group for $i=1,2, \ldots, n$.

(iii) $G$ is a p-group.

(iv) $R / L$ is a finite field of characteristic $p$.

(v) The series $0=G_{0} \leqq G_{1} \leqq \cdots \leqq G_{n}=G$ is a central series.

Proof. (i) Let $\bar{g}$ be a nonzero element of $G_{i} / G_{i-1}$. As $\bar{g} R=G_{i} / G_{i-1}, G_{i} / G_{i-1} \simeq$ $R / \operatorname{Ann}_{R}(\bar{g})=R / L$ and $R$-modules.

(ii) This follows because $R / L$ is a near-field [6, Corollary 2.11] and because the additive group of a finite near-field is an elementary abelian $p$-group.

(iii) is now immediate.

(iv) Since $R / L \leqq \operatorname{End}\left(G_{i} / G_{i-1}\right)$, End $\left(G_{i} / G_{i-1}\right)$ is a ring, and $R / L$ is a near-field, $R / L$ must be a finite division ring which is a field and the characteristic is $p$ by (i) and (ii).

(v) Since $G$ is a $p$-group and $G_{i}$ is a normal subgroup of $G,\left[G_{i} / G_{i-1}, G / G_{i-1}\right]<$ $G_{i} / G_{i-1}$. Thus $\left[G_{i} / G_{i-1}, G / G_{i-1}\right]=0$ since $G_{i} / G_{i-1}$ is a simple $R$-module and the result follows.

We next obtain some information about the group of automorphisms lying in a tame endomorphism near-ring that is local.

Theorem 2.3. Suppose $R$ is a tame endomorphism near-ring of $G$ that is local. Let $A=\operatorname{Aut}(G) \cap R,(R / L)^{*}=R / L-\{0\}$, and $\delta: A \rightarrow(R / L)^{*}$ be the multiplicative homomorphism obtained by restricting the natural projection from $R$ onto $R / L$ to $A$.

(i) $\operatorname{ker} \delta$ is the $p$-Sylow subgroup $P$ of $A$ where $p$ is the prime dividing $|G|$.

(ii) $P$ has a complement $K$ in $A$ which is cyclic and $|K|$ divides $p^{n}-1$ where $p^{n}=|R / L|$.

Proof. (i) First note that if $\alpha \in A$ has order a power of $p$, then $\alpha \delta=1$ since $\left|(R / L)^{*}\right|=$ $p^{n}-1$ is relatively prime to $p$. Conversely suppose $\alpha \in \operatorname{ker} \delta$ and consider an $R$-principal series $0=G_{0} \leqq G_{1} \leqq \cdots \leqq G_{n}=G$ of $G$. Since $1-\alpha \in L$ and $L=\operatorname{Ann}_{R}\left(G_{i} / G_{i-1}\right), \alpha$ stabilizes this series and hence $|\alpha|$ is a power of $p$ by Lemma 1.3. The result now follows.

(ii) This follows from the Schur-Zassenhaus Theorem [1, p. 221] and from the fact that a multiplicative subgroup of finite order in a field is cyclic.

\section{Some local endomorphism near-rings}

We begin this section with an elementary group theory result whose proof we include for the sake of completeness. 
Lemma 3.1. Let $G$ be a p-group, $A$ a p-group of automorphisms of $G$, and $H$ a minimal $A$-invariant subgroup of $G$. Then $A$ acts trivially on $H$.

Proof. Let us momentarily switch to the usual group theory conventions of writing $G$ as a multiplicative group and indicating the action of an automorphism $\alpha$ of $G$ by exponentiation (i.e., $g \alpha=g^{\alpha}$ for $g \in G$ ). Let $[H, A]$ be the subgroup of $G$ generated by the commutators $[h, \alpha]=h^{-1} h^{\alpha}, h \in H, \alpha \in A$. Since $[h, \alpha]^{\beta}=\left[h^{\beta}, \alpha^{\beta}\right]$ for $\beta \in A$, it follows that $[H, A]$ is an $A$-invariant subgroup of $G$. Viewing $[H, A]$ in the semidirect product $G A$ which is also a $p$-group, we have $[H, A]<H$ and hence $[H, A]=1$ since $H$ is a minimal $A$-invariant subgroup.

We now come to the main result of this section. In the proof of this result we shall make use of the socle series of $G$ for a tame endomorphism near-ring $R$ on $G$ which is obtained as follows: The socle of $G$, $\operatorname{Soc}(G)$, is the sum of the minimal $R$-subgroups of $G$ and the socle series is defined by letting $\operatorname{Soc}_{1}(G)=\operatorname{Soc}(G)$ and $\operatorname{Soc}_{k}(G)$ be the inverse image of $\operatorname{Soc}\left(G / \operatorname{Soc}_{k-1}(G)\right)$ in $G$ for $k>1$. By Theorem 10.37 of [7] we have that $\operatorname{Soc}_{n}(G)=G$ for some positive integer $n$.

Theorem 3.2. Let $A$ be a p-group of automorphisms of a p-group $G$ with $\operatorname{Inn}(G) \leqq A$. If $R$ is the endomorphism near-ring of $G$ generated by $A$, then $R$ is local.

Proof. Let

$$
0<\operatorname{Soc}_{1}(G)<\operatorname{Soc}_{2}(G)<\cdots<\operatorname{Soc}_{n}(G)=G
$$

be the socle series of $G$. We first show that $A$ stabilizes this series. By induction on $|G|$, it suffices to show that $A$ acts trivially on $\operatorname{Soc}(G) \operatorname{since}_{R} / \operatorname{Ann}_{R}(G / \operatorname{Soc}(G)$ ) will be an endomorphism near-ring of the same type on $G / \operatorname{Soc}(G)$. But this needed trivial action on $\operatorname{Soc}(G)$ follows from the previous lemma since $\operatorname{Soc}(G)$ is the direct sum of the minimal $R$-subgroups of $G$.

We now have that $1-\alpha$ annihilates the socle series of $G$ for all $\alpha \in A$ and hence $1-\alpha \in J(R)$ by Lemma 2.5 of [4]. Also, $p \cdot 1$ annihilates the socle series since the socle summands are elementary abelian p-groups [7, Theorem 10.30]. Thus it follows that $R / J(R) \simeq Z_{p}$ and $J(R)$ is a maximal right $R$-subgroup of $R$.

By Theorem 2.8 of [6], the proof will be complete if we can show that $J(R)$ is the unique maximal $R$-subgroup of $R$. Let $M$ be a maximal $R$-subgroup of $R$. If $p^{k}$ is the exponent of $G$, then $p^{k} r=0$ for all $r \in R$ and hence the additive group of $R,(R,+)$, is a p-group. Let $N$ denote the normal closure of $(M,+)$ in $(R,+)$; that is, $N$ is the smallest normal subgroup of $(R,+)$ containing $M$ and $N$ is generated by elements of the form $-r+m+r$ where $r \in R, m \in M$. We have that $N<R$ since the normal closure of a proper subgroup of a $p$-group is proper (apply Theorem 4.3.2. of [2], for example). Also if $\alpha \in A$, $r \in R$, and $m \in M$,

$$
(-r+m+r) \alpha=-r \alpha+m \alpha+r \alpha \in N
$$

and it follows that $N$ is an $R$-subgroup of $R$. Thus $N=M$ and $M$ is a right ideal of $R$ [7, Corollary 9.22]. Hence we have that $J(R) \leqq M$ (Theorem 5.17 of [7]) and consequently $J(R)=M$ completing the proof. 
Using Theorems 2.2 and 3.2, we can now characterize those groups $G$ for which $I(G)$ is local. For if $I(G)$ is local, then $G$ is a p-group by Theorem 2.2. Conversely, if $G$ is a p-group, then $I(G)$ is local by Theorem 3.2. Thus we have:

Corollary 3.3. $I(G)$ is local if and only if $G$ is a p-group.

We conclude this section by noting that our results unify some of the work that has already been done on endomorphism near-rings. Specifically, when $G$ is a dihedral 2group or a generalized quaternion group we have an alternative approach for obtaining some of the results in [3] and [5].

If $G$ is a dihedral group of order $2^{n}(n>2)$,

$$
G=\left\langle a, b \mid 2^{n-1} a=2 b=0,-b+a+b=-a\right\rangle,
$$

then $\operatorname{Aut}(G)$ is a 2-group (Lemma 2.2 of [3]) and so it follows that $A(G)$ and $I(G)$ are both local by Theorem 3.2. Moreover, we see from the proof of Theorem 3.2 that $R / J(R) \simeq Z_{2}$ in both cases, thereby obtaining the result of Theorem 3.4 of [3] in this setting. We also remark that $E(G)$ is not local since the projection from $G$ onto the cyclic subgroup generated by $b$ is an idempotent endomorphism of $G$ and local nearrings have no nontrivial idempotents [6, Theorem 4.2].

Similar results hold for $A(G)$ and $I(G)$ if

$$
G=\left\langle a, b \mid 2^{n-1} a=0,2^{n-2} a=2 b,-b+a+b=-a\right\rangle
$$

is a generalized quaternion group of order $2^{n}$ when $n>3$ since Aut $(G)$ is a 2-group [5, Theorem 2]. Moreover these results extend to $E(G)$ since $A(G)=E(G)$ as shown in [5] (Theorem 5) or which can be seen by examining the endomorphisms of $G$ as follows: End $(G)$ has three nontrivial endomorphisms that are not automorphisms whose kernels are the normal subgroups of $G$ generated by $a, b$ and $a+b$ and whose images are the subgroup generated by $2^{n-2} a$ which is the centre of $G$. (See the proof of Theorem 2 of [5]). If $\beta$ is such an endomorphism, it is easily checked that the mapping $\alpha$ defined by $g \alpha=g(1+\beta)$ is an automorphism of $G$ and hence $E(G)=A(G)$.

Finally, we point out that $E(G)$ and $A(G)$ are not local if $G$ is the quaternion group of order 8 (a case not covered in [5]) where we will still have $E(G)=A(G)$. This lack of localness follows because $G$ has an automorphism $\alpha$ of order $3[9$, p. 148] which acts trivially on $\langle 2 a\rangle$. Hence, if $E(G)$ were local, $1-\alpha \in J(R)$ by Lemma 2.1 which violates part (i) of Theorem 2.3. This example brings up the question of whether we might have a converse to Theorem 3.2; that is, if $G$ is a p-group and $A$ is a group of automorphisms of $G$ with $\operatorname{Inn}(G) \leqq A$ which generates a local near-ring $R$, is $A$ a $p$-group? As of this writing we have been unable to resolve this question.

\section{REFERENCES}

1. D. Gorenstein, Finite Groups (Harper and Row, New York, 1968).

2. M. HALl, The Theory of Groups (Macmillan, New York, 1959). 
3. C. G. Lyons and J. J. Malone, Finite dihedral groups and d.g. near rings II, Compositio Math. 26 (1973), 249-259.

4. C. G. Lyons and J. D. P. Meldrum, Characterizing series for faithful d.g. near-rings, Proc. Amer. Math. Soc. 72 (1978), 221-227.

5. J. J. Malone, Generalised quaternion groups and distributively generated near-rings, Proc. Edinburgh Math. Soc. 18 (1973), 235-238.

6. C. J. Maxson, On local near-rings, Math. Z. 106 (1968), 197-205.

7. J. D. P. Meldrum, Near-Rings and Their Links with Groups (Pitman, London, 1985).

8. P. Schmid, Über die stabilitätsgruppen der untergruppenreihen einer endlichen gruppe, Math. Z. 123 (1971), 318-324.

9. H. J. Zassenhaus, The Theory of Groups, second edition (Chelsea, New York, 1958).

Department of Mathematics and Computer SCience

JAMES MADISON UNIVERSITY

HARrisonburg, VA., 22807

U.S.A. 